\title{
EFECTOS DE TANINOS CONDENSADOS DE Schinopsis balansae EN LA DIETA DE CABRAS LECHERAS SOBRE PARÁMETROS PRODUCTIVOS
}

\author{
Martinez, G. M. ${ }^{1}$; Suarez, V. H. ${ }^{2}$; Olmos, L. H. ${ }^{2}$; Alfaro, R. J.1; \\ Alfaro, E. J. ${ }^{1} ;$ Yanes, R. P. ${ }^{1} \&$ Orosco, S. M. ${ }^{2}$
}

\begin{abstract}
RESUMEN
La utilización de taninos condensados de quebracho (TCQ) puede resultar beneficiosa, inocua o perjudicial en el comportamiento productivo de los rumiantes. El objetivo del presente trabajo fue el de evaluar los efectos de la inclusión de TCQ al 2\% del consumo estimado de materia seca (CMS) en la dieta de cabras lecheras sobre el consumo, la producción y la composición de leche. Se emplearon 16 cabras Saanen en inicio de lactancia. El periodo experimental fue de 11 semanas. Los tratamientos fueron: sin suministro de taninos condensados de quebracho (STQ) y con suministro (CTQ). El diseño experimental fue un diseño completamente al azar con mediciones repetidas en el tiempo. El análisis estadístico de los datos se realizó a través del programa InfoStat versión 2017p. No se registraron diferencias estadísticamente significativas para ninguno de los parámetros productivos considerados. Por lo que es posible concluir que la inclusión de TCQ en las condiciones y dosis empleada no reportó beneficio alguno en los parámetros evaluados.
\end{abstract}

Palabras clave: taninos, quebracho, cabras, comportamiento productivo.

\begin{abstract}
Effects of condensed tannins of Schinopsis balansae in dairy goat's productive performance.

The inclusion of quebracho condensed tannins (TCQ) can be beneficial, innocuous or harmful in ruminant's productive performance. The aim of these work was to evaluate the effects on intake, milk production and composition of TCQ inclusion in dairy goat's diet at a concentration of $2 \%$ of estimated dry matter intake (CMS). Sixteen Saanen goats in early lactation were used. The ex-
\end{abstract}

1.- Instituto Nacional de Tecnología Agropecuaria EEA Salta. Ruta Nacional 68 - km 172. (4403) Cerrillos, Salta. E-mail: martínez.gabriela@inta.gob.ar

2.- INTA - Instituto de Investigación Animal del Chaco Semiárido. Área de Salud Animal.

Manuscrito recibido el 21 de diciembre de 2018 y aceptado para su publicación el 30 de mayo de 2019.

Martínez, G. M.; Suárez, V. H.; Olmos, L. H.; Alfaro, R. J.; Alfaro, E. J.; Yanes, R. P. \& Orosco, S. M. Efectos de taninos condensados de Schinopsis balansae en la dieta de cabras lecheras sobre parámetros productivos. FAVE - Ciencias Agrarias 18 (1): 7-14. CC BY-NC-SA 4.0 (c) (i) (-) 


\section{G. M. Martínez et al.}

perimental period was 11 weeks. The treatments were: no supply of quebracho condensed tannins (STQ) and with supply (CTQ). The experimental design was a completely randomized design with measurements repeated over time. Infostat program, 2017p has been used to carry out the statistical analysis. No statistically significant differences were registered for any of the productive parameters considered. So it is possible to conclude that TCQ inclusion in the conditions and dose used did not report any benefit in the parameters evaluated).

Key words: tannins, quebracho, goats, productive performance.

\section{INTRODUCCIÓN}

Los taninos son compuestos fenólicos bioactivos ampliamente distribuidos en el reino vegetal especialmente en leguminosas forrajeras originarias de regiones templadas y tropicales. Se diferencian de otros polifenoles por su habilidad para formar complejos, reversibles o no, con proteínas y precipitarlas. Los taninos más comunes en las plantas se clasifican según su estructura, peso molecular y función en dos categorías: hidrolizables y condensados $(13,21)$.

El rol fundamental de los taninos es la defensa contra la herbivoría y el ataque patógeno, y la conservación de la energía del nitrógeno, particularmente en ambientes de baja fertilidad $(9,20)$.

Existen evidencias que la ingesta de taninos puede resultar beneficiosa, inocua o perjudicial para los rumiantes, dependiendo principalmente de la cantidad consumida, la estructura y el peso molecular del compuesto, pero fundamentalmente de los componentes de la dieta, de la especie animal y su estado fisiológico $(15,17,25,28)$.

Entre los efectos benéficos de los taninos condensado (TC) se destacan una menor excreción de nitrógeno urinario $(13,21)$, la reducción en la producción de metano $(5,8,12)$, y efectos antihelmínticos $(4,15)$, mientras que como efecto negativo se citan a la caída en el consumo de materia seca $(7,24,25)$, una disminución en la digestibilidad del alimento principalmente la fracción nitrogenada y la fibra (27) y en el comportamiento productivo de los animales (29).

En cuanto a la concentración TC varios autores han reportado que al ser ésta de entre un $5 \%$ y $10 \%$ del total de la materia seca ingerida (MSI), el consumo y la digestibilidad del alimento se verían disminuidos, mientras que concentraciones de entre $2 \%$ y $4 \%$ de la MSI favorecerían la absorción intestinal de las proteínas, fundamentalmente por una reducción en la proteólisis por parte de la microflora ruminal, a la vez que no resultaría comprometido el desempeño productivo de los animales $(2,9,19,20,21,23,24)$.

Taninos condensados de quebracho (Schinopsis spp.) (TCQ) es en general la forma en la que se comercializa este tipo de aditivos y en la que se incluye en las raciones de rumiantes. Dentro de las ventajas relativas a la inclusión de TCQ en la dieta de rumiantes se han reportado mejoras en la ganancia de peso vivo en bovinos y una disminución en la producción de metano $(6,20)$. Por su parte, Al-Dobaib (3) suplementando con 1 y $2 \%$ de TCQ ovejas alimentadas con heno de alfalfa obtuvo un leve aumento en la síntesis de nitrógeno microbiano, mientras que con dosis del 
Efectos de taninos condensados en la dieta de cabras lecheras

$3 \%$ ésta disminuyó considerablemente. En bovinos de leche, Aguerre y col. (1) manifestaron un incremento en el rendimiento lácteo acompañado con una disminución en el contenido proteico conforme aumentó el porcentaje de TCQ en la ración.

$\mathrm{Si}$ bien en la literatura existe información disponible, aunque escasa, en cuanto a las consecuencias de los TCQ en la alimentación de rumiantes lecheros en producción poco es lo que se conoce respecto de la inclusión de taninos como aditivo en dietas de caprinos lecheros y sus consecuencias productivas $(16,22)$ Es así que el objetivo del presente trabajo fue el de evaluar los efectos de la inclusión de taninos condensados de quebracho colorado (Schinopsis balansae) al 2\% ingesta estimada de materia seca (CMS) en la dieta de cabras lecheras sobre el consumo, la producción y la composición de leche.

\section{MATERIALES Y MÉTODOS}

Se emplearon 16 cabras multíparas raza Saanen con $19 \pm 9$ días en leche. El periodo experimental fue de 11 semanas: una de acostumbramiento y 10 de ensayo. Los tratamientos fueron definidos como sin suministro de taninos condensados de quebracho (STQ) y con suministro de taninos de quebracho (CTQ). Los animales de cada tratamiento fueron alojados en 2 corrales de 4 animales cada uno. La asignación de los animales a cada corral fue realizada de manera tal que el peso promedio fuera similar, a su vez la selección de los animales para el ensayo se llevó adelante en función del desempeño productivo de las últimas dos lactancias.
A lo largo de todo el experimento los animales recibieron la misma dieta: 0,550 $\mathrm{kg}$ MS maíz/animal/día + 3,500 kg MS heno de alfalfa/animal/día + $20 \mathrm{~g}$ de sal mineral /animal/día. La dosis de taninos condensados de quebracho para el grupo CTQ fue de $80 \mathrm{~g} / \mathrm{animal} /$ día (2\% CMS), cabe destacar que la misma fue mezclada con el maíz partido dispuesto en bateas individuales durante el ordeño (6:00 am). El producto MGM-S de UNITAN S.A.I.C.A (Argentina). fue utilizado para agregar los taninos concentrados a la dieta, los cuales se obtienen a partir de la extracción acuosa del duramen del quebracho colorado chaqueño (Schinopsis balansae).

La producción láctea fue medida diariamente de manera individual a través de lactómetros Waikato. Una vez por semana y a lo largo de todo el ensayo se tomaron muestras de leche de cada animal $(50 \mathrm{ml})$ para evaluar su composición química. Las determinaciones se llevaron adelante mediante espectrofotometría IR S /MILKOSCAN (FT 2, Foss, Dinamarca). Dos veces por semana se registró el consumo de cada corral de heno de alfalfa, mientras que el de maíz/maíz-tanino se monitoreó diariamente. A su vez, cada 15 días se pesaron los animales.

El diseño experimental fue un diseño completamente al azar (DCA) con mediciones repetidas en el tiempo. Se consideraron los efectos del tratamiento, el tiempo y la interacción de ambos. La comparación entre medias fue evaluada a través del test de Tukey $(\alpha=0,05)$. El análisis estadístico de los datos se realizó a través del programa InfoStat versión $2017 \mathrm{p} \AA$. 


\section{RESULTADOS Y DISCUSIÓN}

En la tabla 1 se presentan los resultados obtenidos para cada uno de los parámetros productivos evaluados.

La producción de leche promedio si bien no presentó diferencias estadísticamente significativas resultó un $12 \%$ superior en tratamiento STQ. Existen evidencias que los TCQ disminuyen la concentración de ácidos grasos volátiles (11) y en consecuencia la relación acética:propiónica se ve incrementada (18), por lo que es posible que se produzca un efecto negativo sobre el balance energético y consecuentemente sobre la secreción láctea. Un último ensayo (10) para evaluar el efecto de los TCQ al 1 y 3\% en la ración de vacas lecheras de alta productividad sobre variables productivas y eficiencia en el uso del nitrógeno concluyó en que la inclusión de TCQ bajo las circunstancias del ensayo no fue ventajosa.

Aunque en el presente ensayo no se obtuvieron diferencias significativas en ninguno de los parámetros productivos evaluados, cabe destacar que el consumo de ma- teria seca tampoco resultó modificado por la inclusión de taninos de quebracho. Lo que coincide con lo reportado por Hervas y col. (14) quienes al incluir en la ración de ovejas TCQ a razón de 0,5, 1,5 y 3,0 g/kg de peso vivo y por Al-Dobaib (3) al suplementar ovinos con 1 y $2 \%$ de TCQ quienes tampoco reportaron diferencias respecto al consumo de alimentos. Resultados similares fueron observados por Beauchemin $\mathrm{y}$ col. (6) al incluirlos en la dieta de bovinos en una proporción de $20 \mathrm{~g} / \mathrm{kg}$ MS.

En cuanto a la ingesta de maíz y maíz-tanino no se registraron remanentes en ninguno de los muestreos realizados. Cabe destacar que por lo general los efectos negativos a nivel del comportamiento productivo suele ser explicado por una disminución en el consumo de los alimentos, suceso que no se manifestó en el presente trabajo $(2,25)$. Posiblemente la falta de respuesta entre tratamientos pueda ser explicado por el nivel de inclusión de taninos en la dieta, ya que el mismo respondió al mínimo sugerido en la bibliografía como beneficioso para el comportamiento productivo de los animales $(2,20,24)$.

Tabla 1. Parámetros productivos y consumo de materia seca en función al tipo de tratamiento y a lo largo de todo el experimento.

\begin{tabular}{|c|c|c|c|c|c|c|c|}
\hline \multirow{2}{*}{ Variable } & \multirow{2}{*}{$\mathrm{n}$} & \multicolumn{2}{|c|}{ Tratamiento } & \multirow{2}{*}{$\begin{array}{l}\text { Error } \\
\text { Estándar }\end{array}$} & \multicolumn{3}{|c|}{ Efectos } \\
\hline & & CTQ & STQ & & Tratamiento & Tiempo & $\begin{array}{l}\text { Tratamiento } \\
\text { * Tiempo }\end{array}$ \\
\hline $\begin{array}{l}\text { Producción de leche } \\
\text { (L/animal/día) }\end{array}$ & 1120 & 2,68 & 3,02 & 0,28 & 0,412 & 0,4080 & 0,0685 \\
\hline Peso vivo (kg) & 80 & 56,18 & 56,90 & 0,27 & 0,0607 & $<0,0001$ & 0,2222 \\
\hline $\begin{array}{c}\text { Consumo (kg MS/heno } \\
\text { de alfalfa/día) }\end{array}$ & 80 & 2,73 & 2,63 & 0,05 & 0,1083 & 0,0075 & 0,5299 \\
\hline
\end{tabular}


Efectos de taninos condensados en la dieta de cabras lecheras

Los datos de composición de leche obtenidos en el presente ensayo se reportan en la tabla 2.

La cantidad de flujo de proteína desde el rumen hacia el intestino es el principal factor que modula la respuesta productiva de los rumiantes. Frutos y col. (9) y Waghorn (28) reportaron que los TC al ser consumidos en cantidades moderadas pueden reportar efectos benéficos para los animales sin deprimir el consumo, ya que al unirse y formar complejos con proteína de la dieta evitan su degradación ruminal, dando lugar a un aumento del flujo de aminoácidos al intestino delgado y en consecuencia en la magnitud de aminoácidos esenciales que son absorbidos hacia la sangre.

A su vez, cuando se incrementa la absorción intestinal de aminoácidos es de esperarse un incremento en las concentraciones de proteína en leche (24), parámetro que fue similar en ambos tratamientos $(\mathrm{p}=$ 0,068 ), levemente menor en el tratamiento CTQ. Por su parte, Aguerre y col (1) con vacas Holstein manifestaron al incluirlos en un porcentaje de $0,45,0,90$, and $1,80 \%$ de la MS, una disminución lineal en función de la dosis en el contenido proteico de la leche.
Cabe destacar que el contenido de grasa, lactosa, sólidos no grasos, y sólidos totales no se detectaron diferencias estadísticamente significativas. Min y col. (19) reportaron en vacas lecheras un aumento en la producción láctea, también en la concentración de lactosa y proteína en la mitad y final de la lactancia. En contra posición, Grainger y col. (12) evidenciaron una disminución de los tres parámetros en raciones con 163 y 326 g de TCQ.

\section{CONCLUSIONES}

En virtud de la similitud de los resultados de los parámetros productivos entre los tratamientos, es posible inferir que la inclusión de TCQ en las condiciones y dosis empleadas en el ensayo no resultó suficiente para producir un impacto en la respuesta de cabras lecheras. No obstante, es difícil comparar los resultados del presente estudio con otros, ya que la dieta de base y el tipo de taninos concentrados que se incluya y la especie animal pueden tener diferentes interacciones.

Tabla 2. Composición química de la leche en función al tipo de tratamiento y a lo largo de todo el experimento.

\begin{tabular}{|c|c|c|c|c|c|c|c|}
\hline \multirow{2}{*}{ Variable } & \multirow{2}{*}{$\mathrm{n}$} & \multicolumn{2}{|c|}{ Tratamiento } & \multirow{2}{*}{$\begin{array}{c}\text { Error } \\
\text { Estándar }\end{array}$} & \multicolumn{3}{|c|}{ Efectos } \\
\hline & & CTQ & STQ & & Tratamiento & Tiempo & $\begin{array}{c}\text { Tratamiento * } \\
\text { Tiempo }\end{array}$ \\
\hline \% Proteína & 158 & 3,02 & 3,27 & 0,11 & 0,0681 & 0,0352 & 0,4795 \\
\hline$\%$ Grasa & 158 & 3,99 & 4,29 & 0,16 & 0,1699 & 0,1562 & 0,8163 \\
\hline$\%$ Lactosa & 158 & 4,54 & 4,51 & 0,12 & 0,8166 & 0,0170 & 0,8233 \\
\hline \% Sólidos no grasos & 158 & 8,29 & 8,51 & 0,35 & 0,3805 & 0,0062 & 0,7288 \\
\hline \% Sólidos totales & 158 & 12,28 & 12,82 & 0,19 & 0,2174 & 0,0278 & 0,7768 \\
\hline
\end{tabular}

Revista FAVE - Ciencias Agrarias 18 (1) 2019 | 


\section{AGRADECIMIENTOS}

Los autores quieren hacer llegar su agradecimiento al Ariel López Mato de UNITAN S.A.I.C.A. por la provisión sin cargo de los taninos concentrados.

\section{BIBLIOGRAFÍA}

1.- Aguerre, M. J.; CAPOzZOLO, M. C.; LENCIONI, P.; CABRAL, C. Y WATTIAUX, M. A. 2016. Effect of quebracho-chestnut tannin extracts at 2 dietary crude protein levels on performance, rumen fermentation and nitrogen partitioning in dairy cows. Journal of Dairy Science 99:4476-4486.

2.- AHNERT, S.; DICKHOEFER, U.; SCHULZ, F. Y SUSENBETH, A. 2015. Influence of ruminal Quebracho tannin extract infusion on apparent nutrient digestibility, nitrogen balance, and urinary purine derivatives excretion in heifers. Livestock Science 177: 63-70.

3.- AL-DOBAIB, S.N. 2009. Effect of different levels of quebracho tannin on nitrogen utilization and growth performance of Na-jdi sheep fed alfalfa (Medicago sativa) hay as a sole diet. Animal Science Journal 80: 532541.

4.- ALONSO, M.A.; TORRES, J.F.; SANDOVAL, C.A.; AGUILAR, A.J. Y HOSTE, $\mathrm{H}$. 2008. In vitro larval migration and kinetics of exsheathment of Haemonchus contortus larvae exposed to four tropical tanniniferus plant exctacts. Veterinary Parasitology 153: 313-319.

5.-ANIMUT, G.; PUCHALA, R.; GOETSCH, A.L.; PATRA, A.K.; SAHLU, T.; VAREL, V.H. Y WELLS, J. 2007. Methane emission by goats consuming different source of condensed tannins. Animal Feed Science and Technology 144: 228-241.
6.- BEAUCHEMIN, K.A.; MCGINN, S.M.; MARTINEZ, T.F. Y MCALLISTER, T.A. 2007. Use of condensed tannin extract from Quebracho trees to reduce methane emissions from cattle. Journal of Animal Science 85: 1990-1996.

7.- BEN SALEM, H.; NEFZAOUI, A.; MAKKAR, H.P.S.P.S.; HOCHLEF, H.; BEN SALEM, I. Y BEN SALEM, L. 2005. Effect of early experience and adaptation period on voluntary intake, digestion, and growth in Barbarine lambs given tannin-containing (Acacia cyanophylla Lindl. foliage) or tannin-free (oaten hay) diets. Animal Feed Science and Technology 122: 59-77.

8.- CARULLA, J.E.; KREUZER, M.; MACHMÜLLER, A. Y HESS, H.D. 2005. Supplementation of Acacia mearnsii tannins decreases methanogenesis and urinary nitrogen in forage-fed sheep. Australian Journal Agriculture Research 56: 961-970.

9.- FRUTOS, P.; HERVAS, G.; GIRALDEZ, F.J. Y MANTECON, A.R. 2004. Review. Tannins and ruminant nutrition. Spanish Journal Agriculture Research 2: 191-202.

10.- GERLACH, K.; PRIES, M.; THOLEN, E.; SCHMITHAUSEN, A.J.; BÜSCHER, W. Y SÜDEKUM, K.H. 2018. Effect of condensed tannins in rations of lactating dairy cows on production variables and nitrogen use efficiency. Animal 12 (9): 1847-1855.

11.- GETACHEW, G.; PITTROF F, W.; PUTNAM, D. H.; DANDEKAR, A.; GOYAL, S. Y DEPETERS, E. J. 2008. The influence of addition of gallic acid, tannic acid, or quebracho tannins to alfalfa hay on in vitro rumen fermentation and microbial protein synthesis. Animal Feed Science and Technology 140: 444-461. 
Efectos de taninos condensados en la dieta de cabras lecheras

12.- GRAINGER, C.; CLARKE T.; AULDIST, M. J.; BEAUCHEMIN, K. A.; MCGINN, S. M. Y WAGHORN, G. C. 2009. Potential use of Acacia mearnsii condensed tannins to reduce methane emissions and nitrogen excretion from grazing dairy cows. Canadian Journal Animal Science 89:241-251.

13.- HAGERMAN, A. E. 2002. Tannin handbook. Miami University, Oxford, Ohio. En: http://www.users.muohio.edu/hagermae/ (consultado 9/12/2018).

14.- HERVAS, G.; PEREZ, V.; GIRALDEZ, F. J.; MANTECON, A. R. Y ALMAR MMAND FRUTOS, P. 2003. Intoxication of sheep with quebracho tannin extract. Journal of Comparative Pathology 129:44-54.

15.- HOSTE, H.; TORRES-ACOSTA, J. F. J.; SANDOVAL-CASTRO, C. A.; MUELLER-HARVEY, I.; SOTIRAKI, S.; LOUVANDINI, H.; THAMSBORG, S. M.; TERRILL, T. H. 2015. Tannin containing legumes as a model for nutraceuticals against digestive parasites in livestock. Veterinary Parasitology 212: 5-17.

16.- JERÓNIMO, E.; PINHEIRO, C.; LAMY, E.; DENTINHO, M.T.; SALES-BAPTISTA, E.; LOPES, O. Y CAPELA E SILVA, F. 2016. Tannins in ruminant nutrition: impact on animal performance and quality of edible products. In Tannins: biochemistry, food sources and nutritional properties (ed. CA Combs), pp. 121-168. Nova Science Publishers Inc., NY, USA. En: https://dspace.uevora.pt/rdpc/bitstream/10174/19651/1/ Tannins\%20in\%20Ruminant\%20Nutrition Impact $\% 20$ on $\% 20$ Animal $\% 20$ Performance $\% 20$ and $\% 20$ Quality $\% 20$ of $\% 20$ Edible $\% 20$ Products\%20(author's\%20proof).pdf (consultado 5/12/2018).

17.- LOU, C.N. 1994. Tannin and in vitro digestibility of tropical browse: Predictive equations. Journal of Range Manage 47: 398.
18.- MARTINEZ, T. F.; MCALLISTER, T. A.; WANG, Y. Y REUTER, T. 2006. Effects of tannic acid and quebracho tannins on in vitro ruminal fermentation of wheat and corn grain. Journal of the Science of Food and Agriculture 86: 1244-1256.

19.- MIN, B. R.; BARRY, T. N.; ATTWOOD, G. T. Y MCNABB, W. C. 2003. The effect of condensed tannins on the nutrition and health of ruminants fed fresh temperate forages: a review. Animal Feed Science and Technology 106: 3-19.

20.- MIN, B. R.; ATTWOOD, G. T.; MCNABB, W. C.; MOLAN, A. L. Y BARRY, T. N. 2005. The effect of condensed tannins from Lotus corniculatus on the proteolytic activities and growth of rumen bacteria. Animal Feed Science and Technology 121: 45-58.

21.- MUELLER-HARVEY, I. 2006. Unravelling the conundrum of tannins in animal nutrition and health. Journal of the Science of Food and Agriculture 86(13): 2010-2037.

22.- NAUMANN, H. D.; TEDESCHI, L. O.; ZELLER, W. E. Y HUNTLEY, N. F. 2017. The role of condensed tannins in ruminant animal production: advances, limitations and future directions. Revista Brasileira de Zootecnia 46(12): 929-949.

23.- OTERO, M. E HIDALGO, L. 2004. Condensed tannins in temperate forages species: effects on the productivity of ruminants infected with internal parasites (a review). Livestock Research for Rural Development 16(2): 18-36.

24.- PATRA, A.K. Y SAXENA J. 2011 Exploitation of dietary tannins to improve rumen metabolism and ruminant nutrition. Journal of the Science of Food and Agriculture 91:24-37. 
25.- PILUZZA, G.; SULAS, L.; BULLITTA, S.; AHNERT, S.; DICKHOEFER, U.; SCHULZ, F. Y SUSENBETH, A. 2015. Influence of ruminal quebracho tannin extract infusion on apparent nutrient digestibility, nitrogen balance, and urinary purine derivatives excretion in heifers. Livestock Science 177:63-70

26.- PROVENZA, F. D. 1995. Postingestive feedback as an elementary determinant of food preference and intake in ruminants. Journal of Range Management. 48, 2-17.

27.- TIEMANN, T. T.; LASCANO, C. E.; WETTSTEIN, H. R.; MAYER, A. C.; KREUZER, M. Y HESS, H. D. 2008. Effect of the tropical tannin-rich shrub legumes $\mathrm{Ca}$ lliandra calothyrsus and Flemingia macrophylla on methane emission and nitrogen and energy balance in growing lambs. Animal 2: 790-799.

28.- WAGHORN, G. 2008. Beneficial and detrimental effects of dietary condensed tannins for sustainable sheep and goat production-Progress and challenges. Animal Feed Science and Technology 147: 116-139.

29.- YISEHAK, K.; DE BOEVER, J. L. Y JANSSENS, G. P. J. 2014. The effect of supplementing leaves of four tannin-rich plant species with polyethylene glycol on digestibility and zootechnical performance of zebu bulls (Bos indicus). Journal of Animal Physiology and Animal Nutrition 98: 417-423. 